\title{
Digital Correlator for Measuring the Velocity of Solid Surfaces
}

\author{
Rüdiger Zeitler
}

\begin{abstract}
Correlation techniques offer a good possibility for contactless measurement of the velocity of solid surfaces. The system presented consists of an optical sensor unit, which transforms the stochastic roughness of the object into two signals with a delay time $\tau^{*}$, and of a correlation unit, which extracts the delay time from the signals and calculates the velocity of the surface. The main algorithm of that digital correlator, representing an identification problem of the delay time $\tau^{*}$, is realized by a closed-loop with the advantage of estimating only one point of the correlation function. The dynamic response is mainly dependent on the bandwidth of the sensor signals and the sampling frequency of the correlator, but not on its arithmetic speed. The mathematical description of the correlator is discussed both in the time-domain and the $z$-domain. Based on a linearized system, this allows one to determine the dynamic response and the stability of the closed-loop correlator. Two different applications are presented: the measurement of the velocity of paper webs and the measurement of the length of textile yarns during a winding process.
\end{abstract}

Index Terms-Digital closed-loop correlator, paper web, textile yarn, velocity.

\section{INTRODUCTION}

$\mathbf{T}$ HERE exists a variety of systems for measuring the velocity of solid surfaces. But most of the solutions proposed are developed for a special application or are restricted to a small measurement range. Often, laser Doppler anemometers, spatial frequency methods, or simply wheel encoders are used, but none of them are suitable as a measurement system for series production: laser Doppler anemometers and spatial frequency methods are too expensive while wheel encoders are invasive or measure the velocity with a relatively high systematic error due to slipping effects between the solid surface and the encoder wheel.

The basic idea of transit time correlation to measure the velocity of solid surfaces is well-known in literature [3], [5], [6], but all of the solutions proposed need a difficult signalpreprocessing and/or a high correlator arithmetic speed. It is therefore not possible to develop a low-cost correlation system with high accuracy. As an aim of our current research work an existing field correlator has to be simplified in order to integrate the whole system within one application specific integrated circuit (ASIC) without difficult analog signal-preprocessing.

Manuscript received June 3, 1996.

The author is with the Institute of Measurement and Control, University of Karlsruhe, 76128 Karlsruhe, Germany (e-mail: zeitler@mrt.mach.unikarlsruhe.de).

Publisher Item Identifier S 0018-9456(97)06469-3.

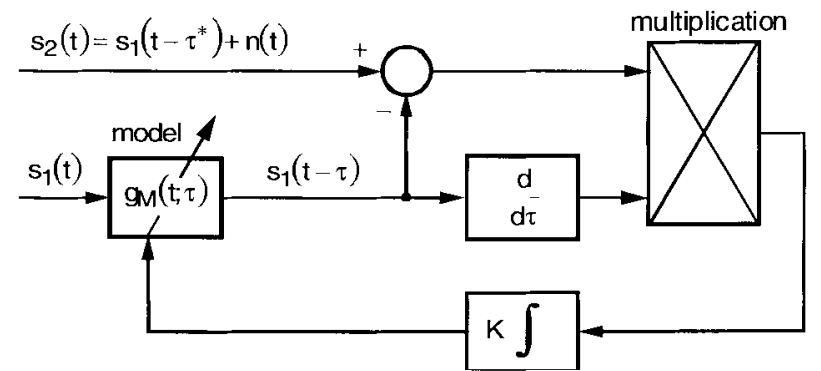

Fig. 1. Principle of a closed-loop correlator.

The principle of that field correlator is shown in Fig. 1. It interprets the process generating the signals $s_{1}(t)$ and $s_{2}(t)$ of two identical sensors placed at a distance $l$ as a linear system of the transport delay type with a delay time $\tau^{*}$. The correlator implements a model of the system and changes its parameter-the model delay time $\tau$-until the difference between the sensor signal $s_{2}(t)$ and the delayed signal $s_{1}(t-\tau)$ is minimal in the mean square sense.

A necessary condition for the minimization is [3]

$$
\frac{d}{d \tau} \mathrm{E}\left\{s_{1}(t-\tau) s_{2}(t)\right\}=\dot{\Phi}_{12}(\tau)=-\Phi_{12}(\tau)=0
$$

where $\dot{\Phi}_{12}(\tau)$ denotes the derivative of the correlation function of the two sensor signals at the position $\tau$ and $\Phi_{12}$ denotes the correlation function of the differentiated sensor signal $s_{1}$ and the sensor signal $s_{2}$. Equation (1) is interpreted in the block diagram of Fig. 1. Obviously, the system calculates only one point of the differentiated correlation function at $\tau$ and uses this value with an integral controller for calculating a new model delay time.

\section{Theory of ThE New Digital Closed-Loop CorRelator}

For easier implementation the sensor signals are usually clipped to one bit with comparators. Therefore the correlator estimates the polarity cross correlation function

$$
\begin{aligned}
R_{12}(\tau) & =\mathrm{E}\left\{s g_{1}(t-\tau) s g_{2}(t)\right\} \\
& =\frac{2}{\pi} \arcsin \frac{\Phi_{12}(\tau)}{\sqrt{\Phi_{11}(0) \Phi_{22}(0)}}
\end{aligned}
$$

with $s g_{1}=\operatorname{sgn}\left[s_{1}\right]$ and $s g_{2}=\operatorname{sgn}\left[s_{2}\right]$. The clipping operation produces slight amplitude distortion of the correlation function, but does not displace the position of its maximum. As the clipped signals and the polarity cross correlation function at its maximum are not differentiable, the differentiation $d / d \tau$ 


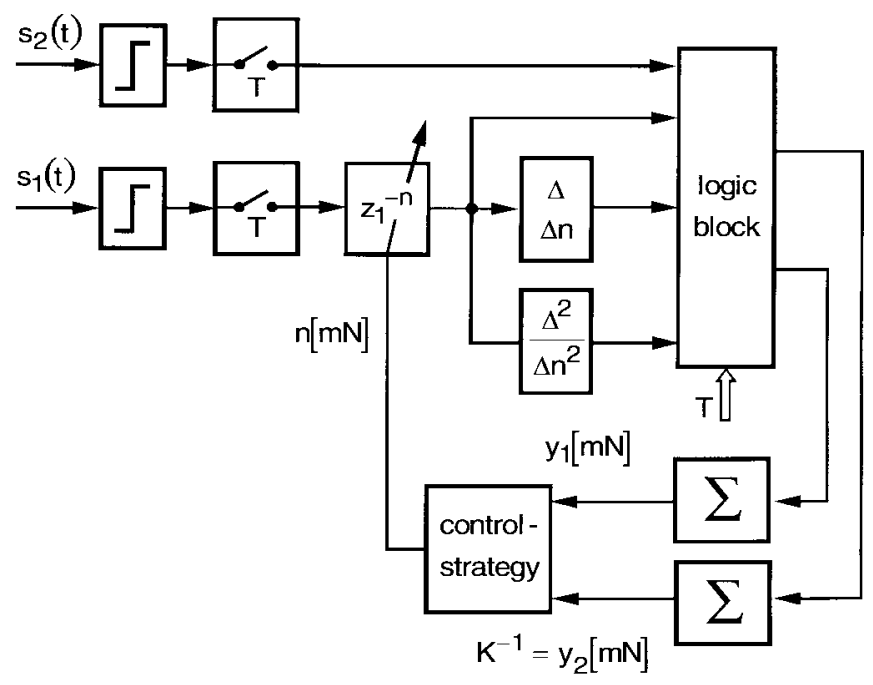

Fig. 2. Principle of the new closed-loop correlator.

in (1) is replaced by a difference quotient $\Delta / \Delta \tau$ of the first clipped signal

$$
\begin{aligned}
\widehat{R}_{12}(\tau) & =\frac{R_{12}(\tau+\Delta \tau)-R_{12}(\tau-\Delta \tau)}{2 \Delta \tau} \\
& =-\mathrm{E}\left\{\frac{s g_{1}(t-\tau+\Delta \tau)-s g_{1}(t-\tau-\Delta \tau)}{2 \Delta \tau} s g_{2}(t)\right\} \\
& =-\mathrm{E}\left\{s \hat{g}_{1}(t-\tau) s g_{2}(t)\right\}
\end{aligned}
$$

with a finite lag difference $\Delta \tau$.

The new principle is shown in Fig. 2. The two sensor signals are high-pass filtered and clipped within the sensor. The sensor signals are sampled with the sampling time $T$ changing the continuous system delay time $\tau^{*}$ to a discrete value $n^{*}$ with

$$
n^{*}=\frac{\tau^{*}}{T} \text {. }
$$

One of the clipped sensor signals is delayed in a RAM in order to generate all necessary signals forming its first and second differences. The logic block combines the binary values of the clipped and sampled signals to estimate the first and second differences of the polarity correlation function $y_{1}[m N]$ and $y_{2}[m N]$. The time averaging with limited time $N T$ replaces the mathematical expectation in (1) and (3). The discrete model delay time

$$
n=\frac{\tau}{T}
$$

is corrected after each averaging according to

$$
n[m N]=n[(m-1) N]+K \cdot y_{1}[(m-1) N] .
$$

The whole system represents a multirate sampling system with a fast sampling time $T$ and a slow sampling time $N T$. The corresponding $z$-domain variables are denoted by $z_{1}$ and $z_{2}$, respectively. An index $k$ refers to $T$ whereas $m$ refers to NT.

With no statistic errors in the steady state, i.e., after a response time greater than the time constant of the system, $n_{m}=n[m N]$ is constant and $y_{1}=0$. Thus, the discrete version of the minimum condition (1) is fulfilled. Equation (6) represents an iterative zero-crossing search algorithm for the function $y_{1}[m N]$.

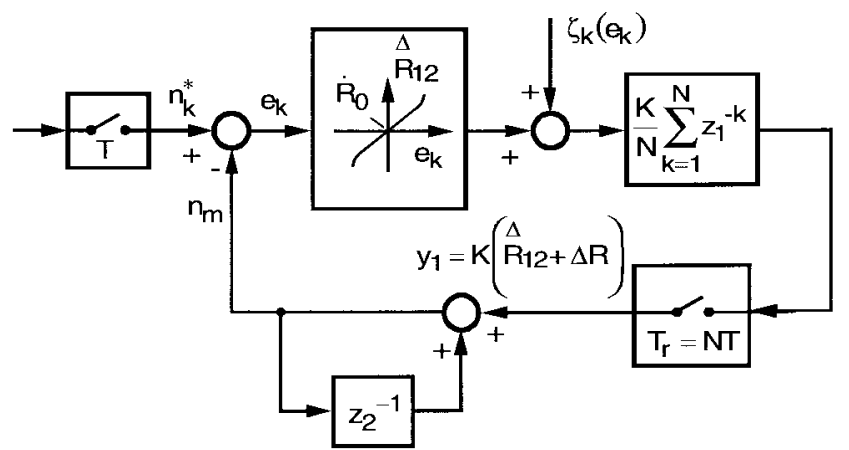

Fig. 3. Equivalent block diagram of the new closed-loop correlator.

The following illustrates why an adaptive gain $K$ is required.

With $K=$ const. $>0$ we obtain the well known gradient algorithm. As the cross correlation function changes with the signal statistics and the velocity [4], it is not possible to set $K=$ const. for a wide measurement range, but $K$ has to be adjusted to the different operating situations. In order to get some helpful hints in choosing $K$, we have to describe the correlator mathematically.

In Fig. 3 a linearized equivalent block diagram of the correlator is shown. $n_{k}^{*}$ denotes the discrete delay time of the process

$$
\dot{R}_{0}=-\left.\frac{d}{d t} \stackrel{\leftrightarrow}{R}_{12}(t)\right|_{t=n^{*} T}
$$

denotes the linearization of $\widehat{R}_{12}$ in the neighborhood of the exact estimate $n=n^{*}$. The input signals $s_{1}$ and $s_{2}$ are replaced by the discrete system delay time $n_{k}^{*}$ and the linearized steadystate characteristics $\dot{R}_{0}$, as both pairs of parameters represent the same dynamic response of the system. In order to represent the stochastic characteristics of the signals $s_{1}$ and $s_{2}$, the noise $\zeta_{k}\left(e_{k}\right)$ is introduced. It is assumed to be a function of $e_{k}$ as the variance of the estimated correlation function and thus the variance of the estimated $\hat{R}_{12}$ depend on $\left(n-n^{*}\right)$, too. The integral controller in Fig. 1 is replaced by simply adding $N$ times the results obtained from the logic block and using a feedback of the model delay time $n_{m}$. For technical surfaces, the extension of the linear region of $\widehat{R}_{12}$ is approximately twice the discrete finite lag difference $\Delta n=\Delta \tau / T$ which can be set to any value $\Delta n<n / 2$, giving the possibility to adjust the dynamic response of the correlator. Substituting $y_{1}[\mathrm{mN}]$ in (6) and using the abbreviation $\dot{Q}_{0}=\dot{R}_{0} \cdot K$, we receive the mathematical description of the correlator in the time domain

$$
\begin{aligned}
n[m N]= & n[(m-1) N] \\
& +\frac{\dot{Q}_{0}}{N} \sum_{k=1}^{N}\left[n^{*}[m N-k]-n[(m-1) N]\right] \\
& +\frac{K}{N} \sum_{k=1}^{N} \zeta_{k}\left(e_{k}\right) \\
= & n[(m-1) N] \\
& +\dot{Q}_{0}\left(\overline{n^{*}}[(m-1) N]-n[(m-1) N]\right) \\
& +K \Delta R \\
n_{m}= & n_{m-1}+\dot{Q}_{0}\left(\overline{n_{m-1}^{*}}-n_{m-1}+\frac{\Delta R}{\dot{R}_{0}}\right)
\end{aligned}
$$



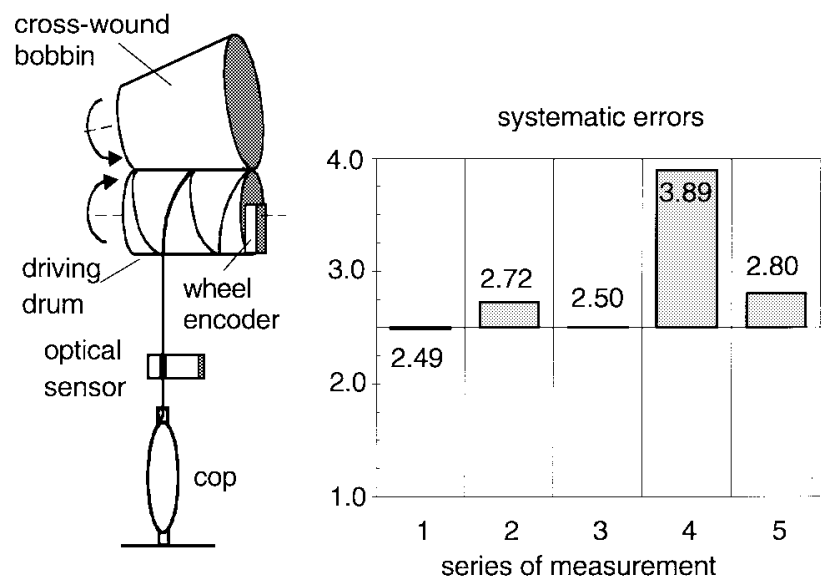

Fig. 4. Typical setup of a winding machine and experimental results changing the parameters of the correlator and the machine.

where $\Delta R$ denotes an abbreviation of the time averaged noise $\zeta_{k}$ of the system and $\overline{n_{m-1}^{*}}$ denotes the mean discrete system delay time over the averaging time $N T$.

By transforming (8), with no statistic errors, that means $\zeta_{k}=0$ and $\Delta R=0$, we obtain the mathematical description of the correlator in the $z$-domain

$$
\tilde{n}\left(z_{2}\right)=\frac{\dot{Q}_{0}}{z_{2}-\left(1-\dot{Q}_{0}\right)} \tilde{n}^{*}\left(z_{2}\right)
$$

where the tilde denotes the $z$-transform. The closed-loop system is stable if the pole $z=1-\dot{Q}_{0}$ has a modulus less than 1 , which is the case for

$$
0<\dot{Q}_{0}<2
$$

With (9), the overall time constant of the closed-loop correlator is given by

$$
T_{c l}=\frac{N T}{-\ln \left|1-\dot{Q}_{0}\right|}
$$

which not only depends on the averaging time $N T$ but also on $\dot{Q}_{0}$.

The systematic error of the correlator is characterized by the incorrect rating

$$
\mu_{m}=\mathrm{E}\left\{\overline{n_{m}^{*}}-n_{m}\right\}
$$

With (8), $\mathrm{E}\{\Delta R\}=0$ and $n_{m}^{*}=$ const. we get

$$
\mu_{m}=\left(1-\dot{Q}_{0}\right) \mu_{m-1}=\left(1-\dot{Q}_{0}\right)^{m-1} \mu_{0}
$$

and with $m \rightarrow \infty, \mu_{\infty}=0$. The correlator works properly, but the transient response depends on the feedback amplification $\dot{R}_{0} \cdot K$. With $\dot{Q}_{0}=1$, i.e., the pole of the return transfer function $z=0$, we obtain the dead-beat-response of the system.

The statistic errors of the closed-loop correlator are represented by the variance

$$
p_{m}=\mathrm{E}\left\{\left[\left(\overline{n_{m}^{*}}-n_{m}\right)-\mu_{m}\right]^{2}\right\}
$$

In [2] it is shown that the variance converges to

$$
p_{\infty}=\frac{\dot{Q}_{0} \frac{b}{\dot{R}_{0}^{2}}}{2-\dot{Q}_{0}\left(1+\frac{a}{\dot{R}_{0}^{2}}\right)}
$$

where the variance of $y_{1}[m N]$ and thus the variance of $\Delta R$, were approximated by a parabola with the extension $a=f(\Delta n)$ and the vertex $b=f(N T, S N R, \Delta n) \propto(N T)^{-1}$. With $a \ll \dot{R}_{0}^{2}$, we get a good estimate for the variance

$$
p_{\infty} \approx \frac{\dot{Q}_{0} \frac{b}{\dot{R}_{0}^{2}}}{2-\dot{Q}_{0}}=\frac{\dot{Q}_{0} \tilde{b}}{N T \dot{R}_{0}^{2}\left(2-\dot{Q}_{0}\right)} .
$$

The statistic errors are mainly dependent on $\dot{Q}_{0}$ but also on the slope of the steady-state characteristics $\dot{R}_{0}$. To get small statistic errors, we need to minimize $\dot{Q}_{0}$ and maximize $\dot{R}_{0}$. In order to get a good dynamic response from the closed-loop with a maximum of $\dot{Q}_{0}$, we must make a compromise between good dynamic response and small statistic errors. A good value is given by $\dot{Q}_{0}=1$ which means $K^{-1}$ should be set close to the slope of $y_{1}[m N]$. This value $K^{-1}=y_{2}[m N]$ (see Fig. 2) must be estimated with the process.

The averaging time of $y_{1}[m N]$ and the sampling frequency can be adjusted to the signal statistics and the velocity, and thus determine the dynamic response of the correlator.

\section{EXPERIMENTAL RESULTS}

A detailed description of the sensor setup for textile yarns is given in [8], the principle of the telecentric sensor setup for measuring the velocity of paper webs is given in [4].

\section{A. Length Measurement at a Winding Machine}

The length measurements of textile yarns were taken at a newly developed industrial-purpose winding machine (see Fig. 4) changing the number of cuts of the yarn from ten to 30 (series 2), the mean velocity of the yarn from $25 \mathrm{~m} / \mathrm{s}$ to $30 \mathrm{~m} / \mathrm{s}$ (series 3), the finite lag difference of the correlator from $\Delta n=n / 4$ to $\Delta n=n / 8$ (series 4 ), and using another sensor (series 5). Five or ten measurements with same parameters are summarized within one series. The reference lengths were determined by afterwards using a special winding machine with low velocity.

Fig. 4 shows the systematic errors for each series of measurement. As the lengths of the yarn are calculated by integration of the velocity over the time of more than $1000 \mathrm{~s}$, the statistic errors of the system can be neglected. Since textile yarn is expandable, a mean systematic error of $2.5 \%$ occurs for all series of measurement (see Fig. 4) because of different loads when winding the yarn and determining the reference lengths with another velocity and therefore with a lower load.

The maximal difference in length from that mean value, except series 4 , is $0.3 \%$ showing the good accuracy of the correlator. Only with series 4 the finite lag difference of the correlator was set to another value resulting in additional 


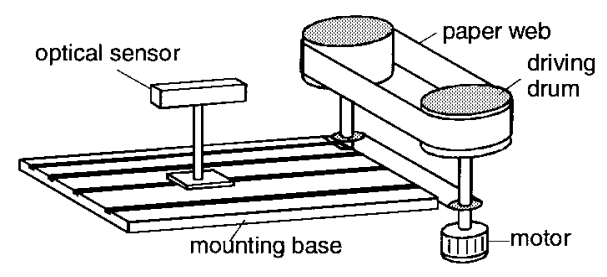

Fig. 5. Test stand for paper webs.

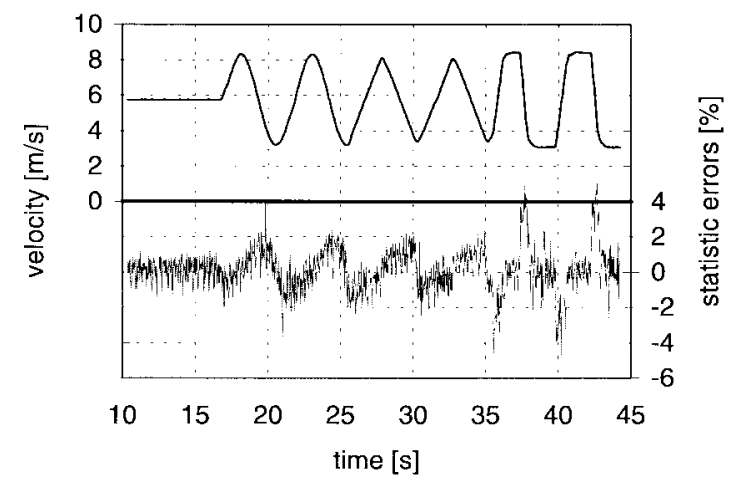

Fig. 6. Test velocity profile of a paper web (top) and error of measurement (bottom).

systematic errors of the correlator. This can be easily explained. During the winding process, the textile yarn rotates resulting in decorrelation of the sensor signals and in an asymmetrical correlation function. Since the correlator uses a difference quotient of the cross correlation function, the zero crossing searching algorithm only detects the peak position of its maximum without error if the function is symmetric to its maximum.

\section{B. Velocity Measurements of Paper Webs}

The measurements were taken at a test stand at the University of Karlsruhe, Germany (Fig. 5). The reference velocity was determined by using a shaft encoder at the driving drum of the test stand.

Fig. 6 shows a test velocity profile of a paper web (top of the figure) and the error of measurement of the correlation system (bottom) at the test stand. The overall time constant $T_{c l}$ of the correlator was set to $32 \mathrm{~ms}$ getting a statistic error of a constant velocity of less than $1 \%$. Three different velocity profiles were set, a sine-function, a sawtooth-function, and a rectangular-function, each with a period of $5 \mathrm{~s}$.

The correlation system follows the given velocity quite well, with an error of less than $2 \%$, due to its good dynamic response. Calculating the maximum slope of the sinewave profile with its period of $5 \mathrm{~s}$ and its amplitude of $2.5 \mathrm{~m} / \mathrm{s}$ we obtain a value of $3.14 \mathrm{~m} / \mathrm{s}^{2}$ but the real value is underestimated since the motor does not follow the exact sinewave profile. This assumption is also confirmed by different statistic errors at the zero crossings of the sinewave profile. A better value of the acceleration limits of the correlator is given with the rectangular profile: with the maximal, constant possible acceleration of the test stand of $6 \mathrm{~m} / \mathrm{s}^{2}$ (edge of the rectangular profile), the measurement error of the correlator rises up to $4.5 \%$.

Next some rough measurements were taken at a paper machine plant at the EFPG in Grenoble, France. These examinations in the wet, press, and dry sections have shown the good functionality of the correlator in difficult environmental conditions, but the accuracy of the system has still to be examined.

\section{CONCLUSIONS}

The new low-cost digital correlator measures the velocity of solid surfaces in a range of $0.5 \mathrm{~m} / \mathrm{s}$ up to $60 \mathrm{~m} / \mathrm{s}$ with a relative error smaller than $1 \%$. Experiments with textile yarns and paper webs at industrial-purpose machines show its good robustness during difficult environmental conditions. The dynamic response of the correlator is well-known and easy to adapt. The newly developed correlator was setup with easy available programmable language devices. In comparison to existing velocity measurement systems, the correlator uses only minimal digital electronics (one full adder, three 1-bit RAM's, one microcontroller, and a few counters), allowing one to integrate the whole system within one ASIC and to setup a system for series production with costs 20 times less than comparable systems.

\section{REFERENCES}

[1] G. Baudin, "Print-through as a quality factor in printing," XII Tech. Conf. Paper Graphic Industry, Budapest, Hungary, 1990.

[2] S. Debatin, "Systematic and statistic errors of one- and two-phase flow measurements with correlation techniques," Ph.D. dissertation, University of Karlsruhe, Germany, 1994.

[3] R. Fritsche and F. Mesch, "Non-contact speed measurement-a comparison of optical systems," Meas. Control, vol. 6, pp. 293-300, 1973.

[4] F. Mesch, "Speed and flow measurement by an intelligent correlation system," in Proc. ISA 90, New Orleans, LA, 1990.

[5] F. Rippinger and G. Schneider, "Low cost self-adaptive correlation measurement system," in Proc. IMEKO World Congr. X, Prague, Czechoslovakia, 1985.

[6] E. Salt, B.L.F. Daku, and H.C. Wood, "Noncontact velocity measurement using time delay estimation techniques," IEEE Trans. Signal Processing, vol. 41, pp. 288-295, Jan. 1993.

[7] R. Zeitler, "Length measurement of spun yarns in the winding process," Melliand Int. Textile Rep., vol. 4, pp. E38-E40, 1996.

[8] _ "Optical sensor for measuring the length of spun yarns," in Proc. GMA Conf. Sensors Measurement Systems, Bad Nauheim, Germany, Mar. 11-13, 1996, pp. 77-82.

Rüdiger Zeitler was born on September 17, 1966, in Rielasingen, Germany. He received the Dipl. Eng. degree in mechanical engineering from the University of Karlsruhe, Karlsruhe, Germany, in 1992. He is currently pursuing the Doctor of Eng. degree at the Institute of Measurement and Control, University of Karlsruhe.

His current research interests include the development of speed and flow measurement systems for industrial purposes. 